\title{
FIGO Stage IIIB
}

National Cancer Institute

\section{Source}

National Cancer Institute. FIGO Stage IIIB. NCI Thesaurus. Code C96257.

A FIGO stage term that applies to gynecologic cancers. For cervical cancer, it refers to cancer that extends to the pelvic wall, and/or causes hydronephrosis or non-functioning kidney; for endometrial cancer, it refers to cancer with vaginal or parametrial involvement. 